\title{
Meeting Report: Dissolution Testing: Current and Future Considerations
}

Mark Santillo ${ }^{1}$, Steve Young ${ }^{2}$, Karin Bracht ${ }^{3}$, David Elder ${ }^{4}$, Axel Zeitler ${ }^{5}$, James Butler ${ }^{6}$, Karl Box ${ }^{7}$, Edmund Kostewicz ${ }^{8}$, James Mann ${ }^{9}$

${ }^{1}$ Plymouth Hospital NHS Trust, Derriford Hospital, Plymouth, Devon, PL6 8DH, UK

${ }^{2}$ British Pharmacopoeia, 151 Buckingham Palace Road, Victoria, London, SW1W 9SZ, UK

${ }^{3}$ MHRA, 151 Buckingham Palace Road, Victoria, London, SW1W 9SZ, UK

4JPAG Chairman, Hertford, Hertfordshire, SG14 2DE, UK

${ }^{5}$ Cambridge University, Cambridgeshire, UK

${ }^{6}$ GlaxoSmithKline, Product Development \& Supply, Park Road, Ware, Hertfordshire, SG12 ODE, UK

7 Sirius Analytical, Forest Row Business Park, Station Road, Forest Row, East Sussex, RH18 5DW, UK

${ }^{8}$ Institute of Pharmaceutical Technology, Goethe University, Frankfurt/Main, Germany

${ }^{9}$ AstraZeneca, Pharmaceutical Technology \& Development, Macclesfield, UK

\section{SYMPOSIUM ORGANIZING COMMITTEE}

The Joint Pharmaceutical Analysis Group (JPAG) and OrBiTo consortium (Oral Biopharmaceutics Tools) ran a highly successful and well-attended symposium covering dissolution testing at the Royal Society of Chemistry, Burlington House, London, the United Kingdom on Thursday 9th February 2017. The symposium was jointly organized by Dr. David Elder (JPAG) and Dr. James Butler (OrBiTo Consortium).

JPAG has over 1,500 members worldwide and is jointly sponsored by the Royal Pharmaceutical Society and the Royal Society of Chemistry, United Kingdom. JPAG's remit is "to encourage, assist and extend the knowledge and study of pharmaceutical analysis and quality control by the holding of scientific meetings, the promotion of lectures, practical demonstrations and discussions".

OrBiTo is part of the EU's IMI (innovative medicines initiative) five year industry/academic collaboration initiative. OrBiTo's objective is to transform our ability to predict the in vivo performance of oral drug products across all stages of drug development. This will be achieved through partnership, collaboration and data sharing. OrBiTo seeks to develop our fundamental knowledge of the gastrointestinal environment to deliver innovative biopharmaceutics tools which will accurately predict product performance over a range of clinically relevant conditions. The integration of in vitro and in silico approaches will provide a biopharmaceutics toolkit, validated using clinical data, to accelerate drug development. Novel, in vivo relevant dissolution tools are a critical component of the toolkit being developed.

\section{INTRODUCTION}

The symposium was chaired by Dr. David Elder, the current JPAG chairman. In his opening remarks, Dr. Elder indicated that dissolution and disintegration were the only release tests for solid oral dosage forms that provide any insight into the rate of release of the drug into the body. Historically, dissolution was viewed primarily as a quality control tool to affirm the rate of release after manufacture and at the end of the product's shelf-life were aligned with global regulatory expectations and commitments. However, over the last two decades, this view has started to change. Now there is clear expectation that dissolution must have clinical relevance and be predictive of any changes that may have an impact on the bioavailability of the product.

Dr. Elder indicated that the objective of this symposium during the morning session was to explore the current state of these techniques from a regulatory, Pharmacopeia, academic, and industry perspective. In the afternoon session, the OrBiTo consortium aimed to explore how emerging biorelevant dissolution tools and media can improve our prediction of oral drug behavior.

\section{MORNING SESSION: \\ CURRENT CONSIDERATIONS}

\section{Dissolution: The Pharmacopoeial Perspective}

Mr. Steve Young (BP/MHRA) delivered the first presentation giving the Pharmacopoeial perspective on dissolution testing. He provided an overview of the role of the Pharmacopoeia in providing the minimum quality standards for drug release throughout the shelf-life of the product. He stressed that Pharmacopeial requirements do not necessarily demonstrate bioequivalence, do not define the QC specification and are not necessarily accepted without question by the regulator. He gave an overview of the British Pharmacopoeia (BP) in terms of notices, chapters, monographs, and appendices and explained how these tied together for dissolution testing.

Mr. Young then pointed out that the BP does not have a specific test for prolonged-release tablets; this is covered 
by a Production Statement that specifies a suitable dissolution test is required (without providing specific details). This is because these preparations are noninterchangeable and often use very different formulation design to control the release rate.

He then outlined the two approaches taken with immediate release preparations, which are the traditional two-stage processes based on $70 \%$ release after 45 minutes, and the harmonized ( $Q$-based) criteria based on a three-stage process and $75 \%$ of label claim released within 45 minutes. There is a decision tree in the supplementary chapter (SC1 E) to inform which limits to employ under different circumstances. Mr. Young discussed recent challenges for the BP with dissolution testing for thyroid hormone preparations and, particularly, levothyroxine tablets where a new, more discriminatory test was introduced in 2014 following reports of issues in switching between different generic products.

Mr. Young finished by highlighting that the BP was undertaking a consultation on dissolution testing that explores whether the approach to dissolution in the BP should now be changed. This can be found at https:// www.pharmacopoeia.com/content/file/ConsultationDissolution-testing-in-BP-finished-products-monographsfor-solid-oral-dosage-forms.pdf (open until 21st April 2017). Users of the Pharmacopoeia are encouraged to provide thoughts and feedback.

Mr. Young was asked about whether higher levels of Stage 2 (or Stage 3) testing of an immediate release product was a compliance issue or whether it was a logical extension of driving increased discrimination into a statistically based test. Mr. Young replied that this was not the intention of the text. A member of the MHRA Licensing Division added that higher levels of testing would not necessarily reflect adversely on the perceived quality of immediate release products, but prolonged release products would receive greater regulatory scrutiny.

\section{Dissolution: The Regulatory Perspective}

Dr. Karin Bracht (MHRA) gave the regulatory perspective on dissolution testing in submissions and outlined the areas of the Common Technical Document (CTD) submission where dissolution testing needs to be referenced. In Method Development (3.2.P.2), the test used must be justified and shown to be robust, reproducible, and discriminatory with regards to critical process parameters and material attributes which will impact bioavailability. She would expect to see data for development batches and a comparison with the target profile in Product and Process Development (3.2.P.2). In Setting Specifications
(3.2.P.5.1 and 5.4), compliance at Stage 2 (of the Q-based method) would be expected for routine manufacturing. Data from the validation batches and for the stability program batches would also be expected in the relevant sections; Dr. Bracht gave an example where changes in the dissolution profile were seen during stability testing and discussed this in relation to the biobatch (the batch used for biopharmaceutical testing) and specification setting.

BCS biowaivers (3.2.P.2) only apply for highly soluble drugs with a rapid or similarly rapid dissolution profile (BCS Class I and III) and only to immediate-release oral preparations (not sublingual/buccal where absorption in the oral cavity occurs). She then raised the issue of ICH M9 covering BCSbased biowaivers which is due to reach Stage 4 by Q2 2019. She gave a comparison of the current requirements of the EU, WHO, Canada, US, and Japan which M9 will set out to harmonize.

Dr. Bracht then went on to describe dissolution testing for other dosage forms and listed some common deficiencies with submissions including a lack of discrimination or a failure to demonstrate discrimination, lack or poorly performed statistical evaluation, specifications that were too wide and not in line with the biobatch, and selection of inappropriate time points.

\section{Dissolution: An Industry Perspective}

Dr. Dave Elder (JPAG/Consultant) provided an industry perspective on dissolution testing. He indicated that dissolution needs to be a robust, reliable technique to ensure the quality of the product, and should be sensitive and bio-discriminatory. He described the evolution of the test and the move to biorelevant test media, newer apparatus which mimic the gastrointestinal tract, and modelling in parallel to give clinically relevant tests and specifications. He discussed some of the considerations such as a single $\mathrm{pH}$ test versus a variable $\mathrm{pH}$, the volume of the vessel, agitation versus peristalsis, etc.

Dr. Elder stressed the differences between QC dissolution methods able to detect deviations in the manufacturing process and biorelevant methods for prediction of IVIVR/ IVIVC; although he did acknowledge that both methods could provide clinically relevant data. He went on to outline some of the issues with current dissolution testing including the position of the dosage form below the paddle and the potential for coning. He questioned why the FDA was introducing a $500 \mathrm{~mL}$ "standard volume" for the test and how there is no historical precedence compared with the extensive data set for larger volumes (typically 900 $\mathrm{mL}$ ). He also discussed whether the same limits could be applied. He raised the issue of using the disintegration test 
as a default release test for BCS I and III substances instead of dissolution testing and finished by asking whether dissolution testing can be reliable and robust as well as sensitive and bio-discriminating.

During $Q \& A$, some attendees questioned whether disintegration would ever be accepted by regulators as a first-intent test during the development phases. Dr. Elder responded that he was aware of some companies who would prefer to use disintegration as the first intent test and subsequently demonstrated that dissolution was not appropriate. This is opposed to the current position, where dissolution is used as the first intent test and companies need to demonstrate that disintegration is more appropriate. He did concede that this was not a universally held position.

\section{DISINTEGRATION: THE FORGOTTEN TEST?}

The final session of the morning was presented by Dr. Axel Zeitler (Cambridge University) and covered disintegration testing asking whether this is the forgotten test. Dr. Zeitler pointed out that rapid disintegration was paramount for a fast therapeutic onset and to maximize bioavailability and also critical for orally dispersible tablets. He stated that the focus on dissolution in industrial practice misses the opportunity to use disintegration testing to try to improve the quality of products. He outlined the complexity of the disintegration process from the medium penetrating into a porous matrix, some of the excipients swelling on hydration and the effect of the medium moving through the matrix. He outlined various equations for understanding liquid transport in a porous matrix but none that could account for the progressive swelling seen with tablets.

Disintegration testing was first defined in 1934, and the test remains largely unchanged; the test is not quantitative and does not report variability. The challenges to developing a more robust test include the wide range of formulations with many excipients showing strongly non-linear swelling. Furthermore, additional processing can have a significant impact on the tablet microstructure that drives disintegration. Previous approaches to try to quantify disintegration have not progressed. More recently, magnetic resonance imaging has been used to look at the disintegration of solid dose preparations. However, there is still a lack of a suitable technique to identify, measure, and quantify the disintegration process.

Dr. Zeitler then discussed a new technique that he has used called "Terahertz Pulsed Imaging," which is a structural imaging procedure (rather than chemical imaging). The spectroscopic method is possible because Terahertz pulses pass through tablets without meaningful absorption; the Dissolution

Technologies AUGUST 2017 more air in the tablet the faster the transmission speed and, hence, the shorter the time of flight. He described some case studies involving Functionalized Calcium Carbonate (FCC), which has an overall porosity up to $65 \%$. Five different tablets with porosities of between $45 \%$ to $65 \%$ were manufactured, and Dr. Zeitler demonstrated that the pore structure could be characterized using Terahertz spectroscopy and x-ray tomography.

A second example of a BCS Class II drug with disintegration times between 5 and 15 minutes was evaluated. This showed no correlation between disintegration time and breaking force (hardness). In contrast, the refractive index at $1 \mathrm{THz}$ showed good correlation with the disintegration time. There was also good correlation with the average dissolution test results, although these results had high variability. Dr. Zeitler outlined how Terahertz imaging can be used to measure liquid wicking and demonstrated how the technique could be used to measure the progress of water through the tablet matrix. He finished by suggesting that disintegration testing could be a valuable technique to speed up batch release.

During the Q\&A session, Dr. Zeitler indicated disintegration, unlike dissolution, could identify poorly wetting compounds. This is important, given that wetting it often a rate-limiting factor for dissolution, even for otherwise highly soluble compounds.

\section{AFTERNOON SESSION: FUTURE CONSIDERATIONS An Overview of IMI OrBiTo and Improved In Vitro Tools for the Prediction of In Vivo Behavior}

The afternoon session commenced with Dr. James Butler (GSK) who presented an overview of an IMI (Innovative Medicines Initiative) OrBiTo initiative for improved in vitro tools for the prediction of in vivo behavior. The vision of OrBiTo was to transform the ability to accurately predict the in vivo behavior of oral drug products across all phases of development. They were looking to develop, validate, and implement innovative tools by defining critical attributes and developing experimental and theoretical models to predict in vivo performance. He outlined the structure of OrBiTo, which is made up of industry partners, academic groups, and SMEs, including a regulatory stakeholder group to ensure acceptance of the outputs in the regulatory environment. He outlined the scope of the five work packages (WPs) covering physicochemical tools, in vitro tools, in vivo tools, in silico models and a management and dissemination package. Dr. Butler is in WP2 developing biorelevant in vitro tools, although there is an overlap into other work packages. He stated that until now, preclinical in vivo animal models were typically more 
trusted for the prediction of human formulation in vivo behavior than biorelevant in vitro tools. Hence, industry needs to move forward.

He stated that OrBiTo was uniquely positioned to use human in vivo studies to inform in vitro tool development from an improved understanding of gastrointestinal dynamics, the impact of food, supersaturation, and precipitation of drugs and permeation at different points in the gastrointestinal tract. This will enable determination of the important parameters for each drug product. WP2 is looking at tools to mimic environments of the gastrointestinal tract, gastrointestinal motility, removal of the drug via permeation, supersaturation and precipitation, and digestion. He then discussed several examples: models to mimic gastric dynamics, USP3 /USP4 models for modified release formulations, the prediction of supersaturation and precipitation, and integrating permeation into the dissolution models. OrBiTo is currently using selected formulations to validate the various options with the aim to build a decision tree for which tool should be used under which circumstances based on formulation type, drug properties, fed/fasted state, etc. This would mark a change from empirical (rule-based) development to rational (model-based) development, with the aim of integrating dissolution data into physiologically based pharmacokinetic (PBPK) models.

\section{Novel Small Scale Predictive Dissolution Methods for Early Stage API Evaluation}

Karl Box (Sirius) then presented on novel small scale predictive dissolution methods for early stage API evaluation. This is part of WP1 and aimed to develop in vitro tools to allow the assessment of biopharmaceutical risk of APIs. Poorly soluble drugs in BCS II and IV classes were selected with a range of acidic, basic, and neutral compounds and measurements of intrinsic dissolution rate (i.e., surface specific dissolution) were performed. Four different media were chosen: FaSSIF v1, pH 6.5 phosphate buffer, Copenhagen FaSSIF, and pH 6.6 MES buffer. Sirius made use of a low volume dissolution platform (T3 PhysChem Platform), other OrBiTo partners used a $\mu$ DISS profiler ( $\mathrm{pION}$ ), both using UV spectrometry for quantification of the drug.

The API was prepared as a $3 \mathrm{~mm}$ compressed disk (using approximately $5 \mathrm{mg}$ of material), thereby presenting only one face with constant surface area to the dissolution medium. However, some OrBiTo partners were using different compression techniques, and there was much variation in the disks and the data. Hence, a uniform method for disk compression was introduced. There were also trials with powdered API, but issues with poor wetting and agglomeration were seen for some samples. Ideally, an independent measurement of particle size is required to calculate the powder surface area. For highly soluble drugs, powder interferences can affect UV spectroscopy with large sample weights, strongly absorbing compounds may also significantly reduce light reaching the detector; however, for poorly soluble compounds, the use of powder can give better data than disc due to a larger surface area and an increased UV response from the dissolving compound. Uppsala University has published guidelines based on solubility: if greater than $1 \mathrm{mg} / \mathrm{mL}$, a disc should be used, if less than $100 \mu \mathrm{g} / \mathrm{mL}$, powder should be used. Sizes that fall between these solubilities may use either option.

Karl Box stated that there was ongoing work with suspensions with a controlled and narrow particle size distribution (typically approximately 1 micron) where the surface area available to the medium was much higher, and consequently, more rapid dissolution was seen. Hence, it would seem that suspensions offer a good alternative for poorly soluble compounds: it is easy to introduce the sample by injecting a known, small quantity of stable suspension and wetting and agglomeration are not an issue. The use of controlled suspensions would also reduce the time needed to run the test. Furthermore, dissolution measurements on suspensions could be used as a link to understanding in vivo performance in preclinical species (e.g., during dose escalation studies).

The results of the different studies showed that many of the poorly soluble drugs had improved dissolution performance in the biorelevant media (e.g., FaSSIF v1). He then pointed out a recent study by the University of Strathclyde in which a four-fold order of magnitude change in solubility can be seen in different variations of clinically relevant intestinal fluid with different bile acid/ phospholipid/oleate ratios. Hence, there is an ongoing discussion on the use of several media, in addition to FaSSIF, to cover the range of expected dissolution behavior when drugs show sensitivity to phospholipid and bile acid content.

He finished the presentation by discussing combined dissolution and permeation equipment using a biphasic dissolution system with a lipid layer in the vessel and looking at distribution between the aqueous and lipid phases. 


\section{In Vitro Methods for Predicting Supersaturation/ Precipitation In Vivo}

Dr. Edmund Kostewicz (Goethe University, Frankfurt) then presented on in vitro methods for predicting supersaturation and precipitation in vivo, introducing the concept of a 'spring and parachute' approach to the generation and maintenance of supersaturation in the gastrointestinal lumen to enhance oral absorption of poorly soluble drugs. The spring is to generate supersaturation and the parachute to retard precipitation; this will be beneficial for drug absorption. He stated that prediction of supersaturation and precipitation is a complex process depending on the physicochemical characteristics of the $\mathrm{API}$, the formulation and the prevailing physiology of the gastrointestinal tract. It is, in fact, challenging to predict based on the API properties alone.

Dr. Kostewicz discussed three models for in vitro tools: (i) a transfer model, (ii) a biorelevant gastrointestinal transfer system (BioGIT), and (iii) an artificial stomach/ duodenum system. The transfer model was based on a two compartment physiologically relevant modified USP dissolution method, the donor (stomach) using FaSSGF-v2 $\mathrm{pH} 2$ and the acceptor (intestine) FaSSIF-v2 pH 6.5. The important parameters include the gastric emptying rate, the intestinal bile salts concentration, the $\mathrm{pH}$, the intestinal volume, and the paddle speed. He discussed an example of ketoconazole (poorly soluble and high permeability, i.e., BCS II), where PBPK modelling was used to integrate the in vitro data and was used to evaluate the influence of supersaturation and precipitation on the absorption characteristics of the drug. His team had used a Stella ${ }^{\circledR}$ 'build your own' physiologically based pharmacokinetic (PBPK) model. He could show that the transfer model could be used to calculate the maximum drug concentration, the precipitation rate, the degree of supersaturation, the dissolved fraction, the fraction solid/precipitated, the final concentration and the area under the curve. The data could then be integrated into the Stella ${ }^{\circledR}$ model to predict plasma concentrations in humans and to enable the evaluation of the effect of the transfer rate, intestinal volume, bile salt concentration and hydrodynamics on supersaturation and precipitation, and, ultimately, oral drug absorption.

The transfer model does not consider drug absorption and, hence, tends to over-predict precipitation for BCS II APIs, but it may be more reflective for BCS IV APIs. Additionally, for high permeability drugs (BCS I/II), the onset of precipitation may be delayed or may not occur at all. However, by combining the results from the in vitro transfer model with Stella ${ }^{\circledR}$ PBPK modelling, which considers drug absorption, an accurate prediction was observed (as in the case for ketoconazole).

Dr. Kostewicz then discussed the BioGIT method which is composed of an initial $250 \mathrm{~mL}$ volume of FaSSGF in the stomach compartment which is transferred into a duodenal compartment containing a $40 \mathrm{~mL}$ volume of FaSSIF. Throughout the experiment, the volume is kept constant through a continual outflow (i.e., for sample collection). To ensure that $\mathrm{pH}$ and bile salt concentration is maintained, there is a continuous inflow of a concentrated FaSSIF solution from a reservoir compartment. The results from two drugs were presented, albendazole and itraconazole (both BCS II), which were formulated into various suspension formulations. This system enables intraluminal concentrations of the duodenum to be simulated and enables the degree of supersaturation and fraction of dose precipitated to be established. The results from this method show a good correlation with intraluminal concentrations measured for the same formulations following dosing to humans. He stated that in vitro models have become more physiologically relevant which has improved their ability to predict gastrointestinal supersaturation and precipitation accurately, and PBPK modelling may be required to appropriately evaluate the in vivo relevance of the in vitro results.

\section{Experiences of a Collaborative Ring Study-Are Simple Biorelevant Tests Robust and Reproducible?}

Finally, Dr. James Mann (AstraZeneca) presented the experiences of a collaborative "ring study" to determine whether biorelevant dissolution methods can be reproducible and robust. The aim was to compare the techniques currently in use within the industry to new techniques under development within OrBiTo. A shortlist of compounds was made, and the ideal candidate(s) would be more than $10 \%$ dissolved after 30 minutes in FaSSIF with incomplete dissolution in the gastric phase and the first 10 minutes in FaSSIF media. Additionally, the products had to be economical and readily available. They selected ibuprofen $600 \mathrm{mg}$ tablets (Brufen Forte) and zafirlukast $20 \mathrm{mg}$ tablets (Accolate), both immediate release tablets, both weak acids, and both with greater dissolution in FaSSIF than FaSSGF media.

A single-stage biorelevant dissolution test was used with FaSSIF-v1, FaSSGF or the option of FeSSIF in a volume of $500 \mathrm{~mL}$ ( $250 \mathrm{~mL}$ for FaSSGF) and USP II dissolution apparatus at $75 \mathrm{rpm}$. Sampling was at 5, 10, 20, and 30 minutes, and every subsequent 30 minutes, until a plateau was reached. There was also a two-stage protocol with $250 \mathrm{~mL}$ FaSSGF being shifted at 30 minutes with 250 $\mathrm{mL}$ double-strength FaSSIF $\mathrm{pH}$ 7.5. There was a degree 
of variation seen particularly in the type of filters used; most partners used manual sampling with HPLC analysis, whereas some used semi-automated methods or a UV analysis. There was also a variation in numbers of samples taken and in time points.

The results for FaSSIF ibuprofen showed good consistency between the 16 OrBiTo partners; there was one outlier in the data set where no obvious reasons could be found. For the two-stage process with ibuprofen, there was one high outlier due to using the same filter for both stages (this is likely to have significantly impacted results due to retained drug on the filter being washed through with the change in $\mathrm{pH})$. In addition, there were also three lower outlier results explainable by (i) one center not pre-heating the FaSSIF media, (ii) one center used a mini-vessel approach, and (iii) one center with a potentially lower $\mathrm{pH}$. Without these four outliers, the data were very consistent. FeSSIF data also showed good correlation for the three partners who performed it, and one partner demonstrated there was good correlation between FaSSIF prepared using a commercial powder and that made in-house.

Dr. Mann then discussed the zafirlukast study where the FaSSIF data showed good correlation across the 16 OrBiTo collaborators. The two-stage process also showed good correlation with just one low result and this from a site that had a low result with ibuprofen case study. The results seen in these studies were in line with previously reported ring studies using USP 3 calibrator tablets (theophylline). Hence, this study showed reproducible and robust methodology in biorelevant media across the 16 OrBiTo partners. The study also identified various pitfalls so that best practices can be shared for the future improvement of consistency. The study has recently been repeated with a weak base, and the results are being evaluated.

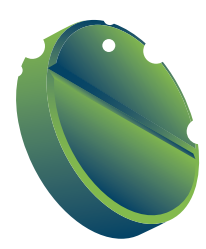

